Serious Gaming to Support Exercise Therapy for Patients with Chronic Nonspecific Low Back Pain: A Feasibility Study.

Peer-reviewed author version

MATHEVE, Thomas; CLAES, Guido; Olivieri, enzo \& TIMMERMANS, Annick (2018) Serious Gaming to Support Exercise Therapy for Patients with Chronic Nonspecific Low Back Pain: A Feasibility Study.. In: Games for Health Journal, 7, p. 1-9 (Art № 4).

DOI: $10.1089 / g 4 h .2017 .0173$

Handle: http://hdl.handle.net/1942/26155 
Serious gaming to support exercise therapy for patients with chronic non-specific low back pain: a

\section{feasibility study}

Running title: Serious gaming for chronic low back pain

Thomas Matheve ${ }^{1}$, Guido Claes $^{2}$, Enzo Olivieri ${ }^{2}$, Annick Timmermans ${ }^{1}$

${ }^{1}$ Rehabilitation Research Center (REVAL), Biomed, Faculty of Medicine and Life Sciences, Hasselt University, Diepenbeek, Belgium; ${ }^{2}$ Department of physical and rehabilitation medicine, Jessa hospital, Hasselt, Belgium.

Thomas Matheve (corresponding author); Annick Timmermans

Hasselt University

Faculty of medicine and life sciences

Agoralaan, building A

3590 Diepenbeek

Belgium

Thomas Matheve: Thomas.Matheve@uhasselt.be; ORCID ID: 0000-0003-2523-9723

Annick Timmermans: Annick.Timmermans@uhasselt.be

Guido Claes; Enzo Olivieri

Department of physical and rehabilitation medicine

Jessa Hospital

Stadsomvaart 11

3500 Hasselt

Belgium

Guido Claes: Guido.Claes@jessazh.be

Enzo Olivieri: Enzo.Olivieri@jessazh.be 


\section{Abstract}

Objective: To investigate the feasibility of a functional exercise program supported by serious gaming for patients with chronic non-specific low back pain.

Materials and Methods: Ten patients with chronic non-specific low back pain and an underlying motor control impairment were recruited. Subjects performed a partially supervised exercise program (36 sessions, 18 weeks) that included 30 minutes of general conditioning and 90 minutes of individually tailored functional motor control exercises. Serious games were used to (1) improve thoracolumbar dissociation and (2) to provide postural feedback during functional motor control exercises. The serious games were also available at home.

Results: Treatment satisfaction and the scores on the credibility/expectancy questionnaire were good and did not change throughout the intervention. Patients remained motivated throughout the rehabilitation program and no serious adverse events were reported. Overall, participants indicated that the serious games helped them to perform the home exercises more correctly, and as a consequence, they felt more confident doing them. However, the time needed to set up the games was a barrier for home-use and participants would have found it useful to receive postural feedback during daily life activities.

Conclusions: It is feasible to support a functional exercise program with serious games for patients with chronic non-specific low back pain, both in a supervised and a home environment. Timeefficiency and the integration of serious games in daily life activities are challenges that need to be addressed in the future.

Keywords: low back pain; serious games; exercise therapy; rehabilitation; feasibility 


\section{Introduction}

Low back pain (LBP) is one of the most common health problems in Western society ${ }^{1}$ and has a substantial impact on daily functioning. ${ }^{1,2}$ Globally, it is the leading cause of disability ${ }^{1}$ and it is one of the most important reasons for work absenteeism. ${ }^{3}$ When the pain persists for more than three months, it is defined as chronic low back pain (CLBP). ${ }^{4}$

Exercise therapy is often the treatment of choice for patients with CLBP. Although this type of intervention has been proven to be effective in reducing pain and disability, the effect sizes are only small to moderate. ${ }^{4,5}$ One of the main reasons for the modest results is that exercise programs are not in line with the current recommendations for exercise therapy, ${ }^{6}$ namely that exercises should be supervised (individually or in group), ${ }^{7}$ integrated in functional tasks, ${ }^{8,9}$ and tailored to the patient's individual needs ${ }^{6-8,10}$ and preferences. ${ }^{7,11,12}$ In addition, the barriers to participate in an exercise program should be addressed. ${ }^{12}$ Examples of these barriers are poor motivation, ${ }^{13-15}$ lack of support during (home) exercises ${ }^{14,15}$ and fear about incorrect exercise performance. ${ }^{12,15}$

Innovative approaches, such as the use of rehabilitation technologies, could potentially overcome some of these barriers. ${ }^{16}$ However, previously investigated technologies show two major weaknesses, namely the lack of gaming aspects (e.g. fun or competition) ${ }^{17}$ and the fact that most technologies were initially developed for other purposes than to support exercise therapy for LBP (e.g. diagnostic ultrasound). ${ }^{16}$ The possible applications of these systems are therefore limited, which implies that they are typically being used during standard exercises in non-functional positions (e.g. transversus abdominis training in lying) ${ }^{18,19}$ Moreover, these technologies cannot be used at home ${ }^{16,20}$ whereas home-based exercises are essential in the rehabilitation of patients with CLBP. ${ }^{21,}$

\footnotetext{
${ }^{22}$ Serious games (SGs) specifically designed for LBP rehabilitation might address the shortcomings of
} 
current technologies. As SGs have the purpose to train new skills or develop new knowledge in a fun and engaging way, ${ }^{17,23}$ they have the potential to increase motivation and adherence, ${ }^{20,24,25}$ which can be an important pathway for the improvement of treatment effects. ${ }^{21,22}$ Secondly, SGs are capable of providing postural feedback in the absence of a therapist and can inform patients about a correct exercise performance, supporting them during their home exercises.

Studies integrating SGs into a tailored and functional rehabilitation program including home exercises are currently lacking for patients with CLBP, in part because most technological systems are not suited to support this type of rehabilitation. ${ }^{16}$ In addition, patients with CLBP typically need to continue exercising for a longer period, ${ }^{11}$ while the motivating effects of SGs might decrease over time. ${ }^{26}$ Hence, it remains questionable whether SGs can be successfully integrated in a long-term rehabilitation program. Therefore, it is worthwhile investigating the feasibility of such an intervention. Accordingly, the primary aims of this feasibility study were: (1) to assess the treatment credibility and expectancy of improvement, (2) to evaluate patients' motivation for a long-term SGsupported exercise program, (3) to assess the feasibility of using SGs at home, and (4) to monitor adverse events. The secondary aim was to evaluate the effectiveness of the program.

\section{Methods}

\section{Participants}

Ten patients who participated in an outpatient rehabilitation program for LBP were recruited at the Jessa Hospital, Belgium. To be included, subjects had to be between 18 and 65 years old, diagnosed with chronic non-specific LBP (> 3 months), and with an underlying motor control impairment. The 
diagnosis of a motor control impairment was based on a comprehensive assessment, which is described elsewhere. ${ }^{27,28}$ Exclusion criteria were: spinal surgery in the past, presence of an underlying serious pathology (e.g. inflammatory diseases), signs or symptoms of nerve root involvement, pregnancy (up to one year postpartum) and an allergy for tape. The study was approved by the medical ethical committees of the Jessa Hospital and of Hasselt University (Hasselt, Belgium). All patients gave written informed consent before being included in the study.

\section{Technological system - serious games}

The ValedoMotion ${ }^{\circledR}$ system (version 1.2, Hocoma, Switzerland) is a rehabilitation tool for patients with LBP. It consists of a laptop, remote control and three inertial wireless motion tracking sensors (40x30x16 mm, $\pm 16 \mathrm{~g})$. Two sensors are mounted to the patient's spine at the L1 and S1 level (Fig. 1), while one sensor is used to calibrate the system. The sensor signals are sent to the laptop, enabling the patient to practice pelvic tilt exercises in a gaming environment (Fig. 2). The system uses the movements of the S1-sensor relative to the L1-sensor to control the games. In this way, patients have to dissociate lumbopelvic movements from the thoracic spine. Secondly, patients can receive feedback during functional motor control exercises (MCEs) using the 'target game' and the 'coconut game'. The target game is displayed as a bull's eye and the coconut game as a tray with coconuts

(Fig. 3). The sensors detect the spinal movements and the cursor on the screen (target game) or the tray (coconut game) will move accordingly. When patients are able to control the lumbopelvic movements, they can keep the cursor in the middle of the bull's eye or prevent the coconuts from falling off the tray. 


\section{Intervention}

A detailed description of the intervention can be found in the Supplementary material. Subjects participated in an outpatient rehabilitation program for LBP that consisted of 36 sessions at a hospital (two hours, twice weekly) and home exercises. Subjects performed a tailored functional exercise program including 30 minutes of general conditioning and 90 minutes of MCEs under partial supervision. During the first three weeks, patients practiced without technological support (standard rehabilitation), after which the technological support was gradually introduced (see Table 1). Patients were asked not to participate in any other form of rehabilitation during the study.

Outcome measures were obtained at baseline and at the end of week $3,8,13$ and 18 (i.e. at the end of the intervention).

\section{Standard rehabilitation}

The general conditioning included cycling on a stationary bike at an intensity of $75 \%$ of the maximal heart rate, and exercises on a stepping machine and crosstrainer.

A summary of the MCE protocol is provided in Table 2. All patients were treated according to these principles, but the exercises were tailored to the patient's specific problem (Fig. 4).

\section{Serious game supported rehabilitation}

The SG-supported exercise therapy was identical to the standard rehabilitation, except that patients received sensor-based postural feedback from the SGs during 45 minutes of MCEs consisting of 
thoracolumbar dissociation exercises and functional MCEs. The rest of the time, patients performed the exercises without feedback.

Thoracolumbar dissociation exercises were trained with SGs that had to be steered with pelvic tilts

(Fig. 2). All the games were played in a standing or in a sitting position, with a duration of two minutes each. Participants played a selection of five games per session. The difficulty level was adjusted for each game throughout the rehabilitation program. First, the games requiring single plane pelvic movements were selected, while the games controlled by 3-dimensional movements were added later.

Regarding the functional MCEs, patients continued their standard rehabilitation, but the exercises were supported by postural feedback from the target/coconut game (Fig. 3). To avoid patients becoming dependent on the feedback and to improve the learning process, the amount of feedback was gradually decreased and eventually omitted during the last five weeks of the intervention. ${ }^{29}$ This is essential, as patients should learn to control their lumbar spine movements during daily life activities when no extrinsic feedback is available.

\section{$\underline{\text { Home exercises }}$}

Participants were given an exercise booklet that contained pictures and a description of the exercises. Between week six and thirteen they received a ValedoMotion ${ }^{\circledR}$ system to support their home exercises. Participants were asked to perform three SGs and three functional MCEs at home, and to implement the principles they learned during daily life activities. 


\section{Outcome measures}

\section{$\underline{\text { Treatment credibility and expectancy for improvement, motivation, treatment satisfaction and }}$}

\section{adherence}

Treatment credibility and the expectancy for improvement were assessed with the credibility/expectancy questionnaire (CEQ) ${ }^{30}$ which consists of the credibility and the expectancy subscales. Both subscales have a total score between 3 and 27, with a higher score reflecting a better result. Training motivation was assessed with the Intrinsic Motivation Inventory (IMI). ${ }^{31}$ The IMI consists out of 35 items divided over of six subscales, with a higher score corresponding with a better outcome (range 1-7). Treatment satisfaction was measured with an 11-point scale $(0=$ not satisfied at all, $10=$ fully satisfied). The adherence towards the treatment program was measured by the number of attended treatment sessions in the hospital (range 1-36).

\section{Feasibility of unsupervised use of the SGs at home}

Therapists recorded the time needed to explain to patients how to use the technological system, the time needed for patients to set up the system themselves, and whether patients were able to place the sensors correctly on the spine.

Using open-ended questions, patients were asked (1) to elaborate on their experiences with the SGs at home, and (2) to indicate how the technological system could be improved. Adherence to home exercises was evaluated with a diary, in which patients were asked to indicate how long they practiced each day, and whether they used the SGs. 


\section{$\underline{\text { Adverse events }}$}

Patients were asked to report any adverse events (e.g. pain flare-ups) to the therapists. Although serious gaming seems to be a safe way of rehabilitation, adverse events are underreported and few studies have used SGs in unsupervised conditions. ${ }^{20,24}$ In addition, experiencing pain during SGs can be a reason for discontinuing the exercises. ${ }^{20}$ Therefore, the number of drop-outs, with reasons why, was recorded.

\section{Effectiveness of the program}

Pain was assessed with the numeric pain rating scale (NPRS). ${ }^{32}$ This is an 11-point scale ranging from 0 to 10 , where patients have to indicate the average intensity of their LBP over the past two days. Disability was assessed with the Roland Morris Questionnaire (RMQ) $)^{33}$ and the patient-specific functioning scale (PSFS). ${ }^{34}$ The RMQ contains 24 questions about the effects of LBP on daily activities, with a higher score (range 0-24) representing a higher level of disability. For the PSFS, the patient has to identify three to five activities that are difficult to perform because of LBP. Each activity is scored on a 0 to 10 scale, with a lower score indicating a higher level of disability. An average score (range 010) was calculated from the scores on the individual activities. Kinesiophobia (i.e. fear of movement) was measured with the Tampa scale for kinesiophobia (TSK). ${ }^{35}$ This questionnaire contains 17 items to assess subjective ratings of kinesiophobia and fear of re-injury due to physical activity. Quality of life was measured with the Short Form-36 Health Survey (SF-36). ${ }^{36}$ The SF-36 consists of 36 items that can be divided into eight subscales and two domains (i.e. a mental and physical health component). A total score for the mental and physical health component was calculated. For the work status assessment, patients had to indicate whether they had a paid job (yes/no), and if so, whether they were on (partial) sick leave because of their LBP (yes/no). 


\section{Statistical Analysis}

Because of the small sample size, non-parametric tests for repeated measures were used. A Friedman analysis was used for continuous data, with a Wilcoxon signed rank test as post-hoc analysis. The Cochran's $Q$ test was used to analyze the dichotomous data. The $\alpha$-level was set at 0.05 , with a Bonferroni correction for the post-hoc tests.

An intention-to-treat analysis was performed by using a single imputation technique for dealing with missing data. The mean proportional change between two test occasions was calculated using the available data for that particular outcome. This proportional difference was used to estimate the missing scores for the subject with missing data.

\section{Results}

Patient characteristics are presented in Table 3. None of the patients received co-interventions during the study.

\section{Treatment credibility and expectancy for improvement, motivation, treatment satisfaction and}

\section{adherence}

An overview of the results is provided in Table 4. Overall, the scores for treatment satisfaction and on the subscales of the CEQ and IMI were moderate to high at baseline, and high at the end of the (SGsupported) intervention. 


\section{Feasibility of unsupervised use of the SGs at home}

It took 20-30 minutes for therapists to explain the system to the patients. After this introduction, all the participants were able to set up and use the system without supervision.

Overall, patients found it positive to have technological support at home. All patients indicated that the postural feedback helped them to perform the exercises more correctly, and as a consequence, they felt more confident doing them. Nine participants considered the SGs to be more motivating and fun than conventional exercises. Six participants reported that towards the end of the rehabilitation, they mainly used the target/coconut game during functional exercises, as they perceived these exercises to be the most useful.

Two main barriers to home use were reported. Although it took only five minutes to set up the system, six patients considered this extra effort as a barrier to use the SGs at home. Secondly, six patients reported that they also preferred to be able to receive postural feedback during daily life activities, such as cleaning or gardening. This would allow them to practice during lunch breaks or job-related tasks.

Because only three participants consistently filled in the home-exercise diary, no conclusions can be drawn regarding the adherence to home exercises.

\section{Adverse events}

One patient reported two episodes of slightly increased pain for several days, but attributed this to a change in working schedule, rather than to the exercises. Apart from a minimal transient increase in pain during exercises, other participants reported no adverse events. One participant dropped out after T1, due to personal reasons, which were not related to the study. 


\section{Effectiveness of the program}

Except for the mental component of the SF-36 $\left(\chi^{2}=1.4, p=0.50\right)$, all other clinical outcomes significantly improved over time. All participants who were on sick leave at baseline had returned to work by the end of the intervention (Table 5).

\section{Discussion}

When offering a new way of rehabilitation, it is valuable to assess the credibility and expectancy of patients towards this approach. Both factors have been shown to be associated with the outcome of a rehabilitation program for patients with CLBP. ${ }^{37,38}$ The results from the CEQ indicate that patients found the SG-supported treatment credible and that they expected the treatment to be effective, and this remained so during the whole intervention. This might be due to the fact that the treatment rationale and the purpose of the SGs were discussed with the patients prior to the start of the intervention. ${ }^{38}$ In addition, participants could probably relate the exercises to their specific impairments because of the functional approach that was based on their personal rehabilitation goals. Patients with LBP value this individual care over a standard intervention, and expect it to be more effective. $^{12,39}$

By integrating SGs into a tailored exercise program, we tried to overcome some important barriers to exercise therapy, such as insufficient support during home exercises, ${ }^{14,15}$ low confidence in a correct exercise performance ${ }^{12,15}$ and poor motivation. ${ }^{14,15,40}$ The participants in our study indicated that they felt supported and more confident about their exercise performance due to the feedback from the SGs at home. With respect to motivation, patients often need extra support (e.g. by a mobile 
app ${ }^{41}$ to continue exercising. ${ }^{42}$ Serious games also have the potential to improve the motivation to exercise, ${ }^{24,25}$ but this has mostly been shown in studies lasting only 4-6 weeks. ${ }^{24,43}$ Because patients with CLBP typically need to exercise for a longer period, ${ }^{11}$ and motivation might decrease over time, ${ }^{26}$ we investigated this during an 18-week intervention. The results from our study showed that patients remained motivated and satisfied throughout the intervention. All of the scores on the subscales of the Intrinsic Motivation Inventory were high at the end of the intervention (>5.3) and none of the scores declined over time. This might explain why the drop-out rate was only $10 \%$ ( 1 patient) and all patients attended $>80 \%$ of the sessions. Unfortunately, the response rates to the home-exercise diaries were very low, so we cannot make any conclusions about the adherence to the home-exercise program, which is a limitation of our study.

Research in patients with musculoskeletal pain shows that it is feasible to integrate SGs into rehabilitation programs. ${ }^{43}$ However, most studies only used supervised exercise programs in hospitals or rehabilitation centers, where the technological system is set up by a therapist. ${ }^{20,43}$ For patients with CLBP, home exercises are an important part of the rehabilitation and, as such, technological support should ideally be provided at home. ${ }^{16}$ Consequently, patients need to be able to use SGs without supervision. Overall, patients from our study found it feasible to use the system at home. However, although it took only several minutes to set up the system, this was sometimes considered as a barrier to use the SGs at home, especially when time to practice was scarce. In addition, most patients would prefer a system that can be used outside the home environment (e.g. at work). This highlights the need for user-friendly rehabilitation technologies that can be used without spatial constraints (e.g. no need for the proximity of a computer). More, in depth qualitative studies pertaining to patients' experiences with unsupervised use of rehabilitation technologies may provide useful information concerning the requirements for future developments of technologies. 
Finally, the small sample size and lack of control group have to be taken into account when interpreting the results of this feasibility study. In particular, the improvements in clinical outcomes should not be overestimated.

\section{Conclusions}

It is feasible and safe to support a long-term and individually tailored functional MCE program with serious games during supervised and home exercises. Patients felt more confident while performing the exercises with postural feedback, found the intervention credible and remained motivated throughout the rehabilitation program. However, these results need to be interpreted with care because of the small sample size and the lack of a control group. Time-efficiency and the integration of serious games in daily life activities are challenges that need to be addressed in the future.

\section{Correspondence}

Thomas Matheve

Agoralaan, Building A - 3590 Diepenbeek, Belgium

Thomas.Matheve@uhasselt.be

\section{Acknowledgements}

The authors would like to thank the physical therapists and patients at the department of physical and rehabilitation medicine of the Jessa Hospital, Belgium. 


\section{Author disclosure statement}

No competing financial interests exist.

\section{Funding}

None.

\section{References}

1. Buchbinder R, Blyth FM, March LM, Brooks P, Woolf AD, Hoy DG. Placing the global burden of low back pain in context. Best practice \& research Clinical rheumatology 2013;27(5):575-89.

2. Hoy D, Bain C, Williams G, March L, Brooks P, Blyth F et al. A systematic review of the global prevalence of low back pain. Arthritis and rheumatism 2012;64(6):2028-37.

3. Dagenais S, Caro J, Haldeman S. A systematic review of low back pain cost of illness studies in the United States and internationally. The spine journal : official journal of the North American Spine Society 2008;8(1):8-20.

4. Hayden JA, van Tulder MW, Malmivaara A, Koes BW. Exercise therapy for treatment of nonspecific low back pain. The Cochrane database of systematic reviews 2005(3):Cd000335.

5. Kamper SJ, Apeldoorn AT, Chiarotto A, Smeets RJ, Ostelo RW, Guzman J et al.

Multidisciplinary biopsychosocial rehabilitation for chronic low back pain: Cochrane systematic review and meta-analysis. Bmj 2015;350:h444.

6. Falla D, Hodges PW. Individualized Exercise Interventions for Spinal Pain. Exercise and sport sciences reviews 2017;45(2):105-15.

7. NICE Guidelines. Low back pain and sciatica in over 16s: assessment and management. Available from: URL: https://www.nice.org.uk/guidance/ng59/chapter/Recommendations.

8. Hodges PW, Van Dillen LR, McGill SM, Brumagne S, Hides JA, Moseley GL. Integrated clinical approach to motor control interventions in low back and pelvic pain. In: Hodges PW, Cholewicki J, Van dieen JH, editors. Spinal Control: The rehabilitation of back pain State of the art and science. 1st ed. London, UK: Churchill Livingstone; 2013. p 243-309.

9. Vibe Fersum K, O'Sullivan P, Skouen JS, Smith A, Kvale A. Efficacy of classification-based cognitive functional therapy in patients with non-specific chronic low back pain: a randomized controlled trial. European journal of pain 2013;17(6):916-28.

10. O'Sullivan K, O'Sullivan P, Vibe Fersum K, Kent P. Better targeting care for individuals with low back pain: opportunities and obstacles. British journal of sports medicine 2017;51(6):489-90.

11. Saragiotto BT, Maher CG, Yamato TP, Costa LO, Costa LC, Ostelo RW et al. Motor Control Exercise for Nonspecific Low Back Pain: A Cochrane Review. Spine 2016;41(16):1284-95.

12. Slade SC, Patel S, Underwood M, Keating JL. What are patient beliefs and perceptions about exercise for nonspecific chronic low back pain? A systematic review of qualitative studies. The Clinical journal of pain 2014;30(11):995-1005. 
13. Demoulin C, Grosdent S, Capron L, Tomasella M, Somville PR, Crielaard JM et al. Effectiveness of a semi-intensive multidisciplinary outpatient rehabilitation program in chronic low back pain. Joint, bone, spine : revue du rhumatisme 2010;77(1):58-63.

14. Beinart NA, Goodchild CE, Weinman JA, Ayis S, Godfrey EL. Individual and interventionrelated factors associated with adherence to home exercise in chronic low back pain: a systematic review. The spine journal : official journal of the North American Spine Society 2013;13(12):1940-50. 15. Palazzo C, Klinger E, Dorner V, Kadri A, Thierry O, Boumenir Y et al. Barriers to home-based exercise program adherence with chronic low back pain: Patient expectations regarding new technologies. Annals of physical and rehabilitation medicine 2016;59(2):107-13.

16. Matheve T, Brumagne S, Timmermans AAA. The Effectiveness of Technology-Supported Exercise Therapy for Low Back Pain: A Systematic Review. American journal of physical medicine \& rehabilitation / Association of Academic Physiatrists 2017;96(5):347-56.

17. Blumberg MF, Burke LC, Hodent PC, Evans MA, Lane HC, Schell J. Serious Games for Health: Features, Challenges, Next Steps. Games for health journal 2014;3(5):270-6.

18. Teyhen DS, Miltenberger CE, Deiters HM, Del Toro YM, Pulliam JN, Childs JD et al. The use of ultrasound imaging of the abdominal drawing-in maneuver in subjects with low back pain. The Journal of orthopaedic and sports physical therapy 2005;35(6):346-55.

19. Unsgaard-Tondel M, Fladmark AM, Salvesen O, Vasseljen O. Motor control exercises, sling exercises, and general exercises for patients with chronic low back pain: a randomized controlled trial with 1-year follow-up. Physical therapy 2010;90(10):1426-40.

20. Skjaeret N, Nawaz A, Morat T, Schoene D, Helbostad JL, Vereijken B. Exercise and rehabilitation delivered through exergames in older adults: An integrative review of technologies, safety and efficacy. International journal of medical informatics 2016;85(1):1-16.

21. Mannion AF, Helbling D, Pulkovski N, Sprott H. Spinal segmental stabilisation exercises for chronic low back pain: programme adherence and its influence on clinical outcome. European spine journal : official publication of the European Spine Society, the European Spinal Deformity Society, and the European Section of the Cervical Spine Research Society 2009;18(12):1881-91.

22. Cecchi F, Pasquini G, Paperini A, Boni R, Castagnoli C, Pistritto $S$ et al. Predictors of response to exercise therapy for chronic low back pain: result of a prospective study with one year follow-up. European journal of physical and rehabilitation medicine 2014;50(2):143-51.

23. Eichenberg C, Schott M. Serious Games for Psychotherapy: A Systematic Review. Games for health journal 2017;6(3):127-35.

24. Bonnechere B, Jansen B, Omelina L, Van Sint Jan S. The use of commercial video games in rehabilitation: a systematic review. International journal of rehabilitation research Internationale Zeitschrift fur Rehabilitationsforschung Revue internationale de recherches de readaptation 2016;39(4):277-90.

25. Swanson LR, Whittinghill DM. Intrinsic or Extrinsic? Using Videogames to Motivate Stroke Survivors: A Systematic Review. Games for health journal 2015;4(3):253-8.

26. Barnett A, Cerin E, Baranowski T. Active video games for youth: a systematic review. Journal of physical activity \& health 2011;8(5):724-37.

27. Dankaerts W, O'Sullivan PB, Straker LM, Burnett AF, Skouen JS. The inter-examiner reliability of a classification method for non-specific chronic low back pain patients with motor control impairment. Manual therapy 2006;11(1):28-39.

28. O'Sullivan P. Diagnosis and classification of chronic low back pain disorders: maladaptive movement and motor control impairments as underlying mechanism. Manual therapy 2005;10(4):242-55.

29. Shumway-Cook A, Woollacott MH. Motor control: translating research into clinical practice. 3rd. ed. Philadelphia: Lippencott, Williams \& Wilkins; 2006.

30. Devilly GJ, Borkovec TD. Psychometric properties of the credibility/expectancy questionnaire. Journal of behavior therapy and experimental psychiatry 2000;31(2):73-86.

31. Ryan RM. Control and information in the intrapersonal sphere: An extension of cognitive evaluation theory. J Pers Soc Psychol 1982;43:450-61. 
32. Von Korff M, Jensen MP, Karoly P. Assessing global pain severity by self-report in clinical and health services research. Spine 2000;25(24):3140-51.

33. Roland M, Morris R. A study of the natural history of back pain. Part I: development of a reliable and sensitive measure of disability in low-back pain. Spine 1983;8(2):141-4.

34. Stratford PW, Gill C, Westaway M, Binkley JM. Assessing disability and change on individual patients: a report of patient-specific measure. Physiotherapy Canada Physiotherapie Canada 1995;47:258-63.

35. Miller RP, Kori SH, Todd DD. The Tampa Scale. Tampa 1991.

36. Ware JEJ, Sherbourne CD. The MOS 36-item short-form health survey (SF-36). I. Conceptual framework and item selection. Med Care 1992;30:473-83.

37. Gurung T, Ellard DR, Mistry D, Patel S, Underwood M. Identifying potential moderators for response to treatment in low back pain: A systematic review. Physiotherapy 2015;101(3):243-51.

38. Smeets RJ, Beelen S, Goossens ME, Schouten EG, Knottnerus JA, Vlaeyen JW. Treatment expectancy and credibility are associated with the outcome of both physical and cognitive-behavioral treatment in chronic low back pain. The Clinical journal of pain 2008;24(4):305-15.

39. Hopayian K, Notley C. A systematic review of low back pain and sciatica patients' expectations and experiences of health care. The spine journal : official journal of the North American Spine Society 2014;14(8):1769-80.

40. Boutevillain L, Dupeyron A. Facilitators and barriers to physical activity in people with chronic low back pain: A qualitative study. 2017;12(7):e0179826.

41. Lambert TE, Harvey LA, Avdalis C, Chen LW, Jeyalingam S, Pratt CA et al. An app with remote support achieves better adherence to home exercise programs than paper handouts in people with musculoskeletal conditions: a randomised trial. J Physiother 2017;63(3):161-7.

42. Jordan JL, Holden MA, Mason EE, Foster NE. Interventions to improve adherence to exercise for chronic musculoskeletal pain in adults. The Cochrane database of systematic reviews 2010(1):Cd005956.

43. Collado-Mateo D, Merellano-Navarro E, Olivares PR, Garcia-Rubio J, Gusi N. Effect of exergames on musculoskeletal pain: A systematic review and meta-analysis. Scandinavian journal of medicine \& science in sports 2017. 